\title{
MOTION PLANNING FOR A CLASS OF BOUNDARY CONTROLLED LINEAR HYPERBOLIC PDE'S INVOLVING FINITE DISTRIBUTED DELAYS
}

\author{
Frank WOITTENNEK ${ }^{1}$ AND JOACHIM RUdOLPH ${ }^{1}$
}

\begin{abstract}
Motion planning and boundary control for a class of linear PDEs with constant coefficients is presented. With the proposed method transitions from rest to rest can be achieved in a prescribed finite time. When parameterizing the system by a flat output, the system trajectories can be calculated from the flat output trajectory by evaluating definite convolution integrals. The compact kernels of the integrals can be calculated using infinite series. Explicit formulae are derived employing Mikusiński's operational calculus. The method is illustrated through an application to a model of a Timoshenko beam, which is clamped on a rotating disk and carries a load at its free end.
\end{abstract}

Mathematics Subject Classification. 35B37, 35L55, 44A40, 93C20.

Received May 21, 2002. Revised March 2, 2003.

\section{INTRODUCTION}

The concept of $\pi$-freeness was introduced within the module theoretic approach to the control of linear delay systems [3]. Using this method, efficient motion planning for those systems is possible $[4,5,14]$ in a way similar to nonlinear flat systems $[1,18]$. The expansion of this approach to more general partial differential equations opened new possibilities to the control of systems governed by partial differential equations.

When applying the flatness based method to parabolic equations, as for instance the heat equation [9] or chemical reactor models $[7,10]$, the solution can be written as convergent series involving flat output derivatives of arbitrary order. Some nonlinear parabolic equations have also been treated [10]. The solution of hyperbolic systems, as for instance simple heat exchanger models [20] or the general telegraph equation [2], can be written using definite convolution integrals, and can, therefore, be interpreted as systems with finite distributed delays and predictions. Hyperbolic systems involving spatially dependent coefficients and nonlinearities have also been studied $[15,16]$.

Although several problems have been solved, there exists no general approach to the solution of the motion planning problem, even in the case of linear PDEs with constant coefficients.

In the present paper, we propose a method which is suitable for performing the motion planning for a class of hyperbolic linear partial differential equations with constant coefficients, controlled by one boundary input. This class is characterized by a hypothesis on the roots of the characteristic polynomial of the associated operational ordinary differential equation (by means of Mikusiński's operational calculus). This allows us to explicitly parameterize solutions corresponding to transitions from rest to rest in finite time, i.e., we show that the system variables can be written as convolution products of the trajectory of a free variable $y$ - the so-called

Keywords and phrases. Flatness, motion planning.

1 Technische Universität Dresden, Germany; e-mail: woittennek@erss11.et.tu-dresden.de,

rudolph@erss11.et.tu-dresden.de 
flat output - and functions with compact support. The transitions from rest to rest can be parameterized by choosing an appropriate trajectory for the flat output $y$. Being able to give an interpretation of the operators and operational functions associated with the convolution kernels we can verify their compact support directly without using the Paley-Wiener theorem. Indeed we get an explicit series expansion formula.

Though in this paper we consider only single input systems, generalization to systems with multiple inputs is possible using the module theoretic approach [4].

The paper is organized as follows. In Section 1 we introduce the class of mathematical models that can be handled with the proposed method. Furthermore, we sketch how the control for a transition from rest to rest can be calculated. In Section 2 some more general results are presented, which are needed in the third section. In the third section, we prove that the method presented in the first section indeed yields a solution of the motion planning problem. Finally, in Section 4, we apply the proposed method to a Timoshenko beam model.

\section{Systems COnsidered And Design Method}

We consider distributed parameter systems in $p$ distributed variables, $w_{1}, \ldots, w_{p}$, depending on time $t$ and on one space variable $x$. A single (spatially lumped) control input $v$ acts on the boundary. More precisely, the system is given as a set of linear homogeneous partial differential equations with constant coefficients:

$$
A w(x, t)=0, \quad x \in \Omega=\left[x_{0}, x_{1}\right] \subset \mathbb{R}, t \in \mathbb{R}^{+}, A \in\left(\mathbb{C}\left[\frac{\partial}{\partial x}, \frac{\partial}{\partial t}\right]\right)^{p \times p}, w(x, t)=\left(w_{1}(x, t), \ldots, w_{p}(x, t)\right)^{T} .
$$

The boundary conditions are

$$
\left.B_{0} w(x, t)\right|_{x=x_{0}}+\left.B_{1} w(x, t)\right|_{x=x_{1}}+C v(t)=0, \quad B_{0}, B_{1} \in\left(\mathbb{C}\left[\frac{\partial}{\partial x}, \frac{\partial}{\partial t}\right]\right)^{N \times p}, C \in\left(\mathbb{C}\left[\frac{\partial}{\partial t}\right]\right)^{N \times 1}, t \in \mathbb{R}^{+}
$$

The following motion planning and control design problem is considered: define a trajectory $\mathbb{R}^{+} \supset\left[0, T^{*}\right] \ni t \mapsto$ $\left(w_{1}(x, t), \ldots, w_{p}(x, t), v(t)\right) \in \mathbb{C}^{p+1}, \forall x \in \Omega$, such that $w_{i}^{(j)}(x, 0)=0, j \geq 0$ and $w_{i}^{(j)}\left(x, T^{*}\right)=0, j>0, i=$ $1, \ldots, p$, i.e., a solution of the above-defined boundary value problem corresponding to a transition from rest to rest in a finite time $T^{*}$. Without loss of generality, the initial conditions are assumed to be zero.

In order to detail the class of pde's considered here, let us assume the following ${ }^{2}$ :

Assumption 1.1. The partial differential operator being the determinant of the matrix $A$ can be written as $L=\operatorname{det} A=\sum_{i+j \leq N} p_{i, j} \frac{\partial^{i}}{\partial x^{i}} \frac{\partial^{j}}{\partial t^{j}}\left(p_{i, j} \in \mathbb{C}\right)$ where $N>0$ is chosen minimally, and $p_{N, 0} \neq 0$. (Note that $N$ is also the number of boundary conditions.)

Denote the symbol of the operator $L$ by $l$, i.e., $l(\lambda, s)=\sum_{i+j \leq N} p_{i, j} \lambda^{i} s^{j}$. Obviously, $L$ can be written as $L=L^{*} L_{2}$, where $L_{2}, L^{*} \in \mathbb{C}\left[\frac{\partial}{\partial x}, \frac{\partial}{\partial t}\right]$, and the symbol $l^{*}$ of $L^{*}$ has the same roots $\lambda_{i}(s), i=1, \ldots, n, n \leq N$ as $l$ but with multiplicity one only. Let $L^{*}=\sum_{i+j \leq n} p_{i, j}^{*} \frac{\partial^{i}}{\partial x^{2}} \frac{\partial^{j}}{\partial t^{j}}$. As a consequence of Assumption 1.1 we have $p_{n, 0}^{*} \neq 0$.

Assumption 1.2. The operator $L^{*}$ is strictly hyperbolic (w.r.t. $\left.x\right)$ (cf. $\left.[8,17]\right)$ i.e. considering its principal symbol

$$
\bar{l}^{*}(\lambda)=\sum_{i+j=n} p_{i, j}^{*} \lambda^{i} s^{j}
$$

\footnotetext{
${ }^{2}$ Though we exclude some trivial cases by Assumptions 1.1 and 1.2, in general we do not a priori verify whether the problem is well posed. If the problem is well posed, we are able to parameterize a set of solutions. Otherwise, at least one of the Assumptions 1.1, 1.2 , or 1.3 does not hold.
} 
the roots $\lambda_{i}(s), i=1, \ldots, n$ are real and distinct for any non-zero $s \in \mathbb{R}$ ( $L^{*}$ has only real distinct characteristics and, moreover, $x=$ const. is not a characteristic).

We interprete the function $[0, \infty) \ni t \mapsto v(t) \in \mathbb{C}$ as an operator in the Mikusiński field of operators ${ }^{3}$ (see the Appendix), $\hat{v} \in \mathcal{M}$. Moreover, $\Omega \times[0, \infty) \ni(x, t) \mapsto w_{l}(x, t) \in \mathbb{C}, l=1, \ldots, p$, is identified with the operational function $\Omega \ni x \mapsto \hat{w}_{l}(x) \in \mathcal{M}$. Derivatives w.r.t. time $t$ can be replaced by powers of the operator $s$ (initial conditions are zero) $[12,13]$. We obtain the ordinary boundary value problem corresponding to (1.1):

$$
\begin{array}{rlrl}
\hat{A} \hat{w}(x) & =0, & & x \in \Omega, \hat{A} \in\left(\mathbb{C}\left[\frac{\partial}{\partial x}, s\right]\right)^{p \times p} \\
\left.\hat{B}_{0} \hat{w}(x)\right|_{x=x_{0}}+\left.\hat{B}_{1} \hat{w}(x)\right|_{x=x_{1}}+\hat{C} \hat{v} & =0, & \hat{B}_{0}, \hat{B}_{1} \in\left(\mathbb{C}\left[\frac{\partial}{\partial x}, s\right]\right)^{N \times p}, \hat{C} \in(\mathbb{C}[s])^{N \times 1} .
\end{array}
$$

Since in the following we always deal with the operational boundary value problem (1.2), for convenience, let us reformulate the Assumptions 1.1 and 1.2 in this context.

Assumption 1.1*. The characteristic polynomial $P$ of the matrix $\hat{A}$ can be written as

$$
P(\lambda)=\sum_{i+j \leq N} p_{i, j} \lambda^{i} s^{j}, \quad p_{i, j} \in \mathbb{C}, \quad p_{N, 0} \neq 0 .
$$

Without loss of generality we assume $p_{N, 0}=1$.

Denote the $\operatorname{roots}^{4}$ of the characteristic polynomial $P$ by $\lambda_{i}, i=1, \ldots, n, n \leq N$, their multiplicities as $\kappa_{i}$. Moreover,

$$
P^{*}(\lambda)=\sum_{j+i \leq n} p_{i, j}^{*} \lambda^{i} s^{j}
$$

is the normalized polynomial having the same roots as $P$ but with multiplicity 1 only.

Assumption $1.2^{*}$. The roots $\bar{\lambda}_{i}, i=1, \ldots, n$ of the polynomial $\bar{P}^{*}(\lambda)=\sum_{i+j=n} p_{i, j}^{*} \bar{\lambda}^{n}$ are real and distinct ${ }^{5}$.

Let us define a family $\mathfrak{P}$ of polynomials $P_{1}, \ldots, P_{\kappa_{\max }}$, where $\kappa_{\max }=\max \left(\kappa_{1}, \ldots, \kappa_{n}\right)$, as

$$
P_{i}(\lambda)=\prod_{j \in I_{i}}\left(\lambda-\lambda_{j}\right)^{\kappa_{j}-i+1}, \quad I_{i}=\left\{j \mid \kappa_{j} \geq i\right\}, \quad i=1, \ldots, \kappa_{\max } .
$$

We denote the degrees of the polynomials $P_{i}$ by $N_{i}$ and the cardinalities of the sets $I_{i}$ as $n_{i}$. Since for every $i=1, \ldots, \kappa_{\max }-1$ the polynomial $P_{i+1}(\lambda)$ is the normalized g.c.d. of $P_{i}(\lambda)$ and $P_{i}^{\prime}(\lambda)$, and $P_{1}(\lambda)=P(\lambda) \in$ $\mathbb{C}[s][\lambda]$, all the polynomials in $\mathfrak{P}$ belong to $\mathbb{C}[s][\lambda]$.

Now we recursively define an adapted linearly independent family $\mathfrak{C}$ of operational functions $\hat{C}_{i, j}: \Omega \rightarrow$ $\mathcal{M}, i=1, \ldots, \kappa_{\max }, j=1, \ldots, n_{i}$ as linear combinations of the operational functions $x^{k-1} \exp \left(x \lambda_{j}\right), j=$ $1, \ldots, n, k=1, \ldots, \kappa_{j}$. The operational function $\hat{C}_{i, 1}$ is the solution of the following initial value problem associated with the polynomial $P_{i}$ :

$$
\sum_{j=0}^{N_{i}} p_{i, j} \hat{C}_{i, 1}^{(j)}(x)=0, \quad \hat{C}_{i, 1}^{(j)}(0)=0, \quad j=0, \ldots, N_{i}-2, \quad \hat{C}_{i, 1}^{\left(N_{i}-1\right)}(0)=1, \quad i=1, \ldots, \kappa_{\max }
$$

\footnotetext{
${ }^{3}$ For a better readability we use the notation $\hat{f}=\{f(t)\}$ and $\hat{g}(x)=\{g(x, t)\}$, respectively.

${ }^{4}$ In [11] it is shown that any equation of the form $\sum_{i=0}^{n} a_{i} \lambda^{i}\left(a_{i} \in \mathbb{C}[s]\right)$ has exactly $n$ (not necessary distinct) roots $\lambda_{1}, \ldots, \lambda_{n} \in \mathcal{M}$.

${ }^{5}$ This is indeed equivalent to Assumption 1.2. (Set $\lambda=s \bar{\lambda}$ in the principal symbol of $L^{*}$.)
} 
where $p_{i, j} \in \mathbb{C}[s]$ denotes the coefficient of $\lambda^{j}$ in the polynomial $P_{i}$. Starting with $\hat{C}_{i, 1}$, for every $i \in\left\{1, \ldots, \kappa_{\max }\right\}$ we can recursively construct another $n_{i}-1$ linearly independent solutions as

$$
\hat{C}_{i, j}(x)=\hat{C}_{i, j-1}^{\prime}(x) \quad\left(j=2, \ldots, n_{i}\right) .
$$

Hence, we have (see Lem. A.3 in the Appendix)

$$
\begin{aligned}
\hat{C}_{i, j}(x) & =\sum_{k \in I_{i}} \sum_{l=0}^{\kappa_{k}-i} x^{l} \hat{F}_{i, j, k, l}(x) \quad \text { with } \quad \hat{F}_{i, j, k, l}(x)=\eta_{i, j, k, l} \exp \left(x \lambda_{k}\right) \\
\eta_{i, j, k, l} & =\frac{1}{\left(\kappa_{k}-i-l\right) ! l !} \frac{\partial^{\kappa_{k}-i-l}}{\partial \lambda_{k}^{\kappa_{k}-i-l}}\left(\frac{\lambda_{k}^{j-1}}{\prod_{m \in I_{i}, m \neq k}\left(\lambda_{k}-\lambda_{m}\right)^{\kappa_{m}}}\right) .
\end{aligned}
$$

The derivatives with respect to $x$ of the operational functions in $\mathfrak{C}$ can be expressed as linear combinations of these functions. Due to their definition this is obvious for the functions $\hat{C}_{i, j}$ with $j<n_{i}$. Furthermore, the definition scheme (1.5) ensures that for every $l \in\{0, \ldots, N-1\}$ there exist $i \in\left\{1, \ldots, \kappa_{\max }\right\}$ and $j \in\left\{1, \ldots, n_{i}\right\}$ such that $\hat{C}_{i, j}^{(k)}(0)=0$ for $k<l, \hat{C}_{i, j}^{(l)}(0)=1$, and $\hat{C}_{i, j}^{(k)}(0) \in \mathbb{C}[s]$ for $k>l$. Therefore, we can express the solution of any initial value problem of the form

$$
\sum_{j=0}^{N} p_{j} \hat{C}^{(j)}(x)=0, \quad \hat{C}^{(j)}(0) \in \mathbb{C}[s], \quad j=0, \ldots, N-1
$$

by a linear combination of the elements in $\mathfrak{C}$ with coefficients in $\mathbb{C}[s]$. Since the derivatives of the functions in $\mathfrak{C}$ are solutions of such an initial value problem, they can be written in this form as well. Thus, $\mathbb{C}[s, \mathfrak{C}]$ is a differential ring with respect to $\frac{\mathrm{d}}{\mathrm{d} x}$.

As a consequence, in $(\mathbb{C}[s, \mathfrak{C}])^{p}$ there exists a fundamental system of solutions $\hat{\mathbf{W}}_{i}=\left(\hat{w}_{i, 1}, \ldots, \hat{w}_{i, p}\right)^{T}(i=$ $1, \ldots, N)$ of the o.d.e. (1.2a). Using the Ansatz

$$
\hat{\mathbf{w}}(x)=\sum_{k=1}^{N} \hat{\mathbf{W}}_{k}(x) \hat{K}_{k}=\hat{W}(x) \hat{\mathbf{K}}
$$

the boundary conditions $(1.2 \mathrm{~b})$ lead to an equation of the form

$$
T(S \hat{\mathbf{K}}+D \hat{v})=0, \quad S \in(\mathbb{C}[s, \mathfrak{D}])^{N \times N}, \quad D \in(\mathbb{C}[s])^{N}
$$

where $\mathfrak{D}$ is the family of operators obtained by evaluating the operational functions in $\mathfrak{C}$ on $\Gamma$ and $T$ is a diagonal matrix with (diagonal) entries $T_{i} \in \mathbb{C}[s, \mathfrak{D}](i=1, \ldots, N)$ being such that the g.c.d. of the elements in the $i$-th row of the matrix $(S, D)$ is a unit in $\mathbb{C}[s, \mathfrak{D}]$.

Assumption 1.3. The boundary conditions (1.2b) are such that $\operatorname{det}(S) \neq 0$.

Introduce a new variable $\hat{y}$, a so-called flat output, via the equations

$$
\hat{v}=\underbrace{\operatorname{det}(S)}_{=: \hat{\mathcal{v}}} \hat{y}, \quad \hat{\mathbf{w}}(x)=\underbrace{\hat{W}(x) S^{\operatorname{adj}} D}_{=: \hat{\mathcal{W}}(x)} \hat{y}
$$

where $S^{\text {adj }}=\operatorname{det}(S) S^{-1}$. With this choice of the flat output $\hat{y}$ equation (1.7) is satisfied.

Let $\mathfrak{C}^{*}$ denote the family of operational functions defined by $\hat{C}_{i, j, \mu}^{*}=s^{\mu} \hat{C}_{i, j}$, where $\mu \leq N_{i}-\kappa_{\max }+i-j$ and $\mathfrak{D}^{*}$ is the family of operators obtained by evaluating the functions in $\mathfrak{C}^{*}$ on $\Gamma$. Replacing the expressions 
of the form $s^{\mu} \hat{C}_{i, j}$ by the elements of $\mathfrak{C}^{*}$ and $\mathfrak{D}^{*}$ in such a way that the degree of the polynomials $\hat{\mathcal{V}} \in \mathbb{C}\left[s, \mathfrak{D}^{*}\right]$ and $\hat{\mathcal{W}} \in \mathbb{C}\left[s, \mathfrak{C}^{*}, \mathfrak{D}^{*}\right]$ in $s$ is minimal we can write this solution (1.8) as

$$
\begin{array}{rlrl}
\hat{v} & =\sum_{j=0}^{\bar{\rho}} \hat{\mathcal{V}}_{j} s^{j} \hat{y}, & \hat{\mathcal{V}}_{j} \in \mathbb{C}\left[\mathfrak{D}^{*}\right] \\
\hat{w}_{i}(x, s) & =\sum_{j=0}^{\tilde{\rho}_{i}} \hat{\mathcal{W}}_{i, j}(x) s^{j} \hat{y}, & \hat{\mathcal{W}}_{i, j}(x) & \in \mathbb{C}\left[\mathfrak{C}^{*}, \mathfrak{D}^{*}\right], i=1, \ldots, p .
\end{array}
$$

This representation is not unique.

It will be shown in Theorem 3.1 below that under the Assumptions $1.1^{*}$ and $1.2^{*}$ on $P$ these formulae give rise to convolution integrals with kernels $\mathcal{V}_{j}$ and $\mathcal{W}_{i, j}$ that have compact support with respect to $t$ (the spatial variable $x$ is interpreted as a parameter) - equivalently, the convolution integrals are definite:

$$
\begin{aligned}
v(t) & =\sum_{j=0}^{\bar{\rho}} \mathcal{V}_{j}(t) \star y^{(j)}(t) \\
w_{i}(x, t) & =\sum_{j=0}^{\tilde{\rho}_{i}} \mathcal{W}_{i, j}(x, t) \star y^{(j)}(t), \quad i=1, \ldots, p .
\end{aligned}
$$

For the parameterization of this solution a trajectory is chosen for the flat output $y$. This is done in such a way that the derivative of order $\max \left(\tilde{\rho}_{1}, \ldots, \tilde{\rho}_{p}, \bar{\rho}\right)$ of this trajectory belongs to the ring $\mathcal{S}$ of piecewise continuous functions $\mathbb{R} \rightarrow \mathbb{R}$ with left-bounded support. Obviously, the trajectories for the distributed variables $w_{i}$ as well as those for the lumped variable $v$ can all be calculated from such a trajectory $[0, \infty) \ni t \mapsto y(t) \in \mathbb{C}$ then. Therefore, we can parameterize transitions from rest to rest by a trajectory $t \mapsto y(t)$ which is constant for $t \geq t^{*}$. The time $T^{*}$ in which the transition is finished depends on the choice of $t^{*}$ and the support of the functions $\mathcal{V}_{j}$ and $\mathcal{W}_{i, j}$. Defining $I_{T}=\left[T^{-}, T^{+}\right]$in such a way that for all $x \in \Omega$ (and all possible $i$ and $j$ ) the support of $\mathcal{W}_{i, j}$ and that of $\mathcal{V}_{j}$ (w.r.t. time) is a subset of $I_{T}$, one has $T^{*} \leq t^{*}+T^{+}-T^{-}$.

\section{Operational FUnCtions LEADING TO COMPACT SUPPORT CONVOLUTION}

Next we provide results that will allow us to interprete the operational functions $\hat{C}_{i, j, \mu}$ (see Eq. (1.6a) for the definition) occurring in the solution formulae.

Lemma 2.1. Let $P(\lambda)$ be a polynomial satisfying Assumption 1.1*. Denote the roots of $P$ as $\lambda_{i} \in \mathcal{M}, i=$ $1, \ldots, n$ their multiplicity with $\kappa_{i}$. Moreover, $P^{*}(\lambda)=\prod_{i=1}^{n}\left(\lambda-\lambda_{i}\right)=\sum_{j+i \leq n} p_{i, j}^{*} \tilde{\lambda}^{i} s^{j+i}$ is the associated polynomial with roots of multiplicity one only. Assume that $P^{*}$ satisfies Assumption 1.2*. Then, the roots of $P$ can be written as $\lambda_{i}=s \tilde{\lambda}_{i}$, where the $\tilde{\lambda}_{i}$ have operationally convergent series expansions

$$
\tilde{\lambda}_{i}=\sum_{j=0}^{\infty} \frac{\psi^{(j)}(0)}{j !} s^{-j \sigma}
$$

with $\psi_{i}: \mathbb{C} \rightarrow \mathbb{C}$ being analytic in 0 .

Proof. Due to Lemma A.1 the analyticity of the $\psi_{i}$ implies the convergence of the above series. Since $P^{*}$ and $P$ have the same roots, we show the analyticity of the $\psi_{i}$ using the polynomial $P^{*}$.

We replace $\lambda$ by $s \tilde{\lambda}$ and $\lambda_{j}$ by $s \tilde{\lambda}_{j}, j=1, \ldots, n$ and obtain $P^{*}(\lambda)=s^{n} \tilde{P}^{*}(\tilde{\lambda})$, which defines $\tilde{P}^{*}(\tilde{\lambda})=$ $\prod_{j=1}^{n}\left(\tilde{\lambda}-\tilde{\lambda}_{j}\right)=\sum_{j+i \leq n} p_{i, j}^{*} \tilde{\lambda}^{i} s^{j+i-n}$. Obviously, the normalized polynomial $\tilde{P}^{*}$ has coefficients in $\mathbb{C}\left[s^{-\sigma}\right]$ for 
appropriate $\sigma \geq 1$ in $\mathbb{N}\left(\sigma=1\right.$ is always possible). As $s$ is not a divisor of zero in $\mathcal{M}$, the equation $s^{n} \tilde{P}^{*}(\lambda)=0$ implies $\tilde{P}^{*}(\tilde{\lambda})=0$.

Replace $s^{-\sigma}$ by an independent variable $\zeta$ and $\tilde{P}^{*}$ by a function $\mathbb{C}[\zeta, \tilde{\lambda}] \ni H(\zeta, \tilde{\lambda})=\sum_{j+i \leq n} p_{i, j}^{*} \tilde{\lambda}^{i} \zeta^{\frac{1}{\sigma}(n-j-i)}$ of two variables $\zeta$ and $\tilde{\lambda}$. Furthermore, replace the $\tilde{\lambda}_{i}$ by functions $\psi_{i}$ (of the independent variable $\zeta$ ) implicitly defined through the equation $H(\zeta, \psi(\zeta))=0$.

According to Assumption $1.2^{*}$, at $\zeta=0$ this equation has $n$ distinct solutions $\psi_{i}(0)=\bar{\lambda}_{i}, i=1, \ldots, n$. Since, as a consequence, $\frac{\partial H}{\partial \lambda}\left(\zeta, \psi_{i}(\zeta)\right)=\prod_{j \in\{1, \ldots, n\} \backslash\{i\}}\left(\psi_{i}(\zeta)-\psi_{j}(\zeta)\right)$ does not vanish in $\left(0, \psi_{i}(0)\right)$, it follows from

$$
\frac{\mathrm{d} \psi_{i}}{\mathrm{~d} \zeta}(\zeta) \frac{\partial H}{\partial \tilde{\lambda}}\left(\zeta, \psi_{i}(\zeta)\right)+\frac{\partial H}{\partial \zeta}\left(\zeta, \psi_{i}(\zeta)\right)=0
$$

that the derivatives of the functions $\psi_{i}, i=1, \ldots, n$ exist. Therefore, they are all analytic in $\zeta=0$.

Lemma 2.2. Let $\lambda_{i}$ be the roots of a polynomial $P$ satisfying the assumptions of Lemma 2.1.

Then, the operators $\eta_{i, j, k, l}$ defined in (1.6b) can be written as $\eta_{i, j, k, l}=s^{-N_{i}+j+l} \tilde{\eta}_{i, j, k, l}$. The (operational convergent) series expansions of the $\tilde{\eta}_{i, j, k, l}$ read

$$
\tilde{\eta}_{i, j, k, l}=\sum_{m=0}^{\infty} \frac{\gamma_{i, j, k, l}^{(m)}(0)}{m !} s^{-m \sigma}
$$

where $\gamma_{i, j, k, l}$ is a function $\mathbb{C} \rightarrow \mathbb{C}$ which is analytic in 0 .

Proof. Using $\lambda_{i}=s \psi_{i}\left(s^{-\sigma}\right)$, from (1.6b) we obtain

$$
\tilde{\eta}_{i, j, k, l}=\gamma_{i, j, k, l}\left(s^{-\sigma}\right)=\left[\frac{1}{\left(\kappa_{k}-i\right) !}\left(\begin{array}{c}
\kappa_{k}-i \\
l
\end{array}\right)\left(\frac{\partial}{\partial \tilde{\lambda}_{k}}\right)^{\kappa_{k}-i-l}\left(\frac{\tilde{\lambda}_{k}^{j-1}}{\prod_{\alpha \in I_{i}, \alpha \neq k}\left(\tilde{\lambda}_{k}-\psi_{\alpha}\left(s^{-\sigma}\right)\right)^{\kappa_{\alpha}-i}}\right)\right]_{\tilde{\lambda}_{k}=\psi_{k}\left(s^{-\sigma}\right)} .
$$

We substitute $s^{-\sigma}$ by the complex variable $\zeta$. Since, according to Lemma 2.1, all the $\psi_{i}$ are analytic in $\zeta=0$ and $i \neq j \Rightarrow \psi_{i}(0) \neq \psi_{j}(0)$ by assumption, the $\gamma_{i, j, k, l}$ are analytic in $\zeta=0$.

Lemma 2.3. The operational functions in $\mathfrak{C}^{*}$ have a formal power series expansion of the form

$$
\hat{C}_{i, j}(x)=\sum_{k=0}^{\infty} \frac{c_{i, j, k}}{k !} x^{k}, \quad c_{i, j, k} \in \mathbb{C}[s] .
$$

Proof. Since, for any $i=1, \ldots, \kappa_{\max }, \hat{C}_{i, 1}$ is defined as the solution of the initial value problem (1.5a), the first $N_{i}-1$ coefficients of its power series are equal to zero, while $c_{i, 1, N_{i}-1}=1 \in \mathbb{C}[s]$. The coefficients $c_{i, 1, k}$ $\left(k \geq N_{i}\right)$ can be obtained from the first $N_{i}$ coefficients using the polynomial $P_{i} \in \mathbb{C}[s, \lambda]$. Applying the recursion formula $C_{i, j+1}(x)=C_{i, j}^{\prime}(x)$ yields the coefficients $c_{i, j, k}=c_{i, 1, k+j-1} \in \mathbb{C}[s]\left(j=2, \ldots, n_{i}, k \in \mathbb{N}\right)$. Therefore, $\hat{C}_{i, j}(x) \in \mathbb{C}[s] \llbracket x \rrbracket\left(i=1, \ldots, \kappa_{\max }, j=1, \ldots, n_{i}\right)$.

Theorem 2.4 (Interpretation of exponential operators). Consider the operational function $\hat{F}: \mathbb{R} \rightarrow \mathcal{M}$ defined by

Furthermore, let

$$
\hat{F}(x)=\eta \exp (x \lambda) \quad \text { with } \quad \lambda=s \tilde{\lambda}, \quad \eta=s^{-\nu} \tilde{\eta}, \quad \nu \in \mathbb{N} .
$$

$$
\tilde{\lambda}=\sum_{j=0}^{\infty} \frac{\psi^{(n)}(0)}{j !} s^{-j \sigma}, \quad \tilde{\eta}=\sum_{j=0}^{\infty} \frac{\gamma^{(j)}(0)}{j !} s^{-j \sigma}, \quad \sigma \in \mathbb{N} \backslash\{0\}
$$

where $\mathbb{C} \ni \zeta \mapsto \psi(\zeta) \in \mathbb{C}$ and $\mathbb{C} \ni \zeta \mapsto \gamma(\zeta) \in \mathbb{C}$ are analytic in $\zeta=0$, and $\psi(0) \in \mathbb{R}$. Since $s^{-\sigma}=$ $\left\{h(t) \frac{t^{\sigma-1}}{(\sigma-1) !}\right\} \in \mathcal{C}(0<\sigma \in \mathbb{N})$, the series (2.4) are both operationally convergent (see Lem. A.1 in the Appendix). 
Under these assumptions the operational function $\hat{F}$ can be identified with a function $F: \mathbb{R}^{2} \rightarrow \mathbb{C}$ given by

$$
F(x, t)=\left\{\begin{array}{lll}
h(t+x \psi(0)) \sum_{k=0}^{\infty} \frac{1}{k !} \tilde{f}^{(k)}(0 ; x, t, \sigma k+\nu-1), & \nu>0 & \\
\delta(t+x \psi(0)) \gamma(0) \mathrm{e}^{x \psi^{\prime}(0)}+h(t+x \psi(0)) \sum_{k=1}^{\infty} \frac{1}{k !} \tilde{f}^{(k)}(0 ; x, t, k-1), & \nu=0, & \sigma=1 \\
\delta(t+x \psi(0)) \gamma(0)+h(t+x \psi(0)) \sum_{k=1}^{\infty} \frac{1}{k !} \tilde{f}^{(k)}(0 ; x, t, \sigma k-1), & \nu=0, & \sigma>1 .
\end{array}\right.
$$

Here $h$ denotes the Heaviside function and $\delta$ is (the analogue of) the Dirac distribution. The function $\tilde{f}: \mathbb{C} \rightarrow \mathbb{C}$ is defined by

$$
\tilde{f}(\zeta ; x, t, \mu)=\frac{\gamma(\zeta)}{\mu !}(x \psi(\zeta)+t)^{\mu},
$$

where $x, t$, and $\mu$ are considered as parameters.

Proof. The operational function $\hat{F}$ defined by (2.3) can be rewritten as

$$
\hat{F}(x)=s^{-\nu} \mathrm{e}^{s x \psi(0)} \tilde{\eta} \mathrm{e}^{s x(\tilde{\lambda}-\psi(0))} .
$$

Since $s(\tilde{\lambda}-\psi(0)) \in \mathcal{C}^{*}\left(\right.$ where $\mathcal{C}^{*}$ is the ring generated by all complex multiples of the unit in $\mathcal{M}$ over $\mathcal{C}$ ), the exponential function can be expanded in an operationally convergent power series in $x$ (see Lem. A.2 in the Appendix):

$$
\hat{F}(x)=\mathrm{e}^{s x \psi(0)} \tilde{\eta} \sum_{n=0}^{\infty} \frac{s^{n-\nu} x^{n}}{n !}(\tilde{\lambda}-\psi(0))^{n} .
$$

The functions $\gamma$ and $\psi$ being analytic in 0 (by assumption), we can rewrite every term of the series using the operationally convergent series expansion of $\tilde{\eta}(\tilde{\lambda}-\psi(0))^{n}$ in $s^{-\sigma} \in \mathcal{C}$ (see Lem. A.1 in the Appendix). As

$$
\left[\frac{\mathrm{d}^{k}}{\mathrm{~d} \zeta^{k}}\left(\gamma(\zeta)(\psi(\zeta)-\psi(0))^{n}\right)\right]_{\zeta=0}=0, \quad \text { for } n>k
$$

the first $n$ series coefficients of every term vanish

$$
\hat{F}(x)=\mathrm{e}^{s x \psi(0)} \sum_{n=0}^{\infty} \frac{s^{n-\nu} x^{n}}{n !} \sum_{k=n}^{\infty} \frac{s^{-\sigma k}}{k !}\left[\frac{\mathrm{d}^{k}}{\mathrm{~d} \zeta^{k}}\left(\gamma(\zeta)(\psi(\zeta)-\psi(0))^{n}\right)\right]_{\zeta=0} .
$$

Changing the order of summation (convergent series), we obtain

$$
\hat{F}(x)=\mathrm{e}^{s x \psi(0)} \sum_{k=0}^{\infty} \frac{1}{k !}\left[\frac{\mathrm{d}^{k}}{\mathrm{~d} \zeta^{k}} \sum_{n=0}^{k} \frac{x^{n} s^{-\sigma k+n-\nu}}{n !}(\psi(\zeta)-\psi(0))^{n} \gamma(\zeta)\right]_{\zeta=0} .
$$

The series can be translated term by term using (see the Appendix) $s^{-i}(i-1) !=\left\{h(t) t^{i-1}\right\}$, $0<i \in \mathbb{N}$ and $\mathrm{e}^{\alpha s} \hat{f}=\{f(t+\alpha)\}, \alpha \in \mathbb{R}$ :

$$
F(x, t)= \begin{cases}h(t+x \psi(0)) \sum_{k=0}^{\infty} \frac{1}{k !}\left[\frac{\mathrm{d}^{k}}{\mathrm{~d} \zeta^{k}} \sum_{n=0}^{k} \frac{1}{n !} \frac{(t+x \psi(0))^{\sigma k+\nu-n-1}}{(\sigma k+\nu-n-1) !}(x \psi(\zeta)-x \psi(0))^{n} \gamma(\zeta)\right]_{\zeta=0}, \quad \nu>0 \\ \frac{\mathrm{d}}{\mathrm{d} t}\left[h(t+x \psi(0)) \sum_{k=0}^{\infty} \frac{1}{k !}\left[\frac{\mathrm{d}^{k}}{\mathrm{~d} \zeta^{k}} \sum_{n=0}^{k} \frac{x^{n}}{n !} \frac{(t+x \psi(0))^{\sigma k-n}}{(\sigma k-n) !}(x \psi(\zeta)-x \psi(0))^{n} \gamma(\zeta)\right]_{\zeta=0}\right], \quad \nu=0 .\end{cases}
$$


Here, in order to interprete the leading exponential function as shift operator, we make use of the assumption $\psi(0) \in \mathbb{R}$. Using formula (2.7), summation in the inner sum can be continued up to $n=\sigma k$ without changing the result. Applying the binomial formula, we can rewrite the sums as powers. After differentiation w.r.t. $t$ in the case $\nu=0$ we obtain the formulae (2.5).

Lemma 2.5. Let the functions $q_{l, i}(\zeta), i=1, \ldots, N(N, l \in \mathbb{N})$, be analytic in $\zeta=0$, and define

$$
Q_{l}(\zeta)=\sum_{i=1}^{N} q_{l, i}(\zeta)
$$

If $c_{l}=s^{l-\nu} Q_{l}\left(s^{-\sigma}\right) \in \mathbb{R}[s]$ with $\nu \in \mathbb{N}, \sigma \in \mathbb{N} \backslash\{0\}$ then $Q_{l}(\zeta) \in \mathbb{R}[\zeta]$ and, moreover,

$$
\begin{cases}Q_{l}(\zeta)=0, & \text { if } \quad l<\nu \\ \sigma \operatorname{deg} Q_{l}(\zeta) \leq(l-\nu), & \text { if } l \geq \nu\end{cases}
$$

Proof. From $c_{l} \in \mathbb{R}[s]$ it follows $Q_{l}\left(s^{-\sigma}\right) \in \mathbb{R}\left[s^{-1}, s\right]$ or equivalently $Q_{l}(\zeta) \in \mathbb{R}\left[\zeta^{-1 / \sigma}, \zeta^{1 / \sigma}\right]$. Since $q_{l, i}(\zeta)$ is analytic in $\zeta=0, Q_{l}(\zeta)$ is analytic in $\zeta=0$, too. It follows $Q_{l}(\zeta) \in \mathbb{R}[\zeta]$. The second conclusion is a consequence of the assumption $c_{l} \in \mathbb{R}[s]$ (the contrary would yield $c_{l} \in \mathbb{R}\left[s, s^{-1}\right]$ ).

Theorem 2.6 (Compact support). Consider

$$
\hat{C}(x)=\sum_{i=1}^{n} \sum_{k=0}^{\kappa_{i}-1} x^{k} \hat{F}_{i, k}(x) \quad \text { with } \quad \hat{F}_{i, k}(x)=\eta_{i, k} \exp \left(x \lambda_{i}\right), \quad \kappa_{i} \in \mathbb{N} \backslash\{0\}
$$

and assume that $\eta_{i, k}=s^{-\nu+k} \tilde{\eta}_{i, k}(\mathbb{N} \ni \nu \geq k)$ and $\lambda_{i}=s \tilde{\lambda}_{i}$, where $\tilde{\eta}_{i, k}$ and $\tilde{\lambda}_{i}$ satisfy the assumptions on $\tilde{\eta}$ and $\tilde{\lambda}$ in Theorem 2.4. If, in addition, the coefficients of the formal power series of $\hat{C}$ in $x$ can be chosen polynomial, i.e., $\hat{C} \in \mathbb{C}[s] \llbracket x \rrbracket$, then the function $C: \mathbb{R} \times \mathbb{R} \rightarrow \mathbb{C}$, defined by $\hat{C}(x)=\{C(x, t)\}$, is supported in

$$
I \subseteq\left\{(x, t) \mid x \in \mathbb{R}, t \in\left[-\max \left(x \psi_{1}(0), \ldots, x \psi_{n}(0)\right),-\min \left(x \psi_{1}(0), \ldots, x \psi_{n}(0)\right)\right]\right\} .
$$

Proof. In the case $t<-\max \left(x \psi_{1}(0), \ldots, x \psi_{n}(0)\right)$ the values of $F_{i, k}(i=1, \ldots, n)$ are equal to zero and $C(x, t)=0$, too. If $t>-\min \left(x \psi_{1}(0), \ldots, x \psi_{n}(0)\right)$, then according to Theorem 2.4 the function $C$ satisfies

$$
C(x, t)=\sum_{i=1}^{n} \sum_{k=0}^{\kappa_{i}-1} x^{k} \sum_{m=\underline{m}}^{\infty} \frac{1}{m !} \tilde{f}_{i, k}^{(m)}(0 ; x, t, \sigma m+\nu-k-1) \quad \text { with } \quad \tilde{f}_{i, k}(\zeta ; x, t, \mu)=\frac{\gamma_{i, k}(\zeta)}{\mu !}\left(x \psi_{i}(\zeta)+t\right)^{\mu},
$$

where $\underline{m}_{\nu, k}=\max (0,1-\nu+k)$. Changing the order of summation, we can rewrite this equation using $\bar{k}_{i, m}=\min \left(\sigma m+\nu-1, \kappa_{i}-1\right)$

$$
C(x, t)=\sum_{m=0}^{\infty} \frac{X_{m}^{(m)}(0 ; x, t)}{m !} \quad \text { with } \quad X_{m}(\zeta ; x, t)=\sum_{i=1}^{n} \sum_{k=0}^{\bar{k}_{i, m}} x^{k} \tilde{f}_{i, k}(\zeta ; x, t, \sigma m+\nu-k-1) .
$$

In $X_{m}$, we substitute $\tilde{f}_{i, k}$ by its definition, $c f .(2.9)$, and apply the binomial formula

$$
X_{m}(\zeta ; x, t)=\sum_{i=1}^{n} \sum_{k=0}^{\bar{k}_{i, m}} \frac{x^{k}\left(t+x \psi_{i}(\zeta)\right)^{\sigma m+\nu-k-1} \gamma_{i, k}(\zeta)}{(\sigma m+\nu-k-1) !}=\sum_{i=1}^{n} \sum_{k=0}^{\bar{k}_{i, m}} \sum_{l=k}^{\sigma m+\nu-1} \frac{t^{\sigma m+\nu-1-l} x^{l}\left(\psi_{i}(\zeta)\right)^{l-k} \gamma_{i, k}(\zeta)}{(l-k) !(\sigma m+\nu-1-l) !} .
$$


Finally, another change of the summation order yields

$$
X_{m}(\zeta ; x, t)=\sum_{l=0}^{\sigma m+\nu-1} \frac{1}{(\sigma m+\nu-1-l) !} t^{\sigma m+\nu-1-l} x^{l} \underbrace{\sum_{i=1}^{n} \sum_{k=0}^{\min \left(l, \kappa_{i}-1\right)} \frac{1}{(l-k) !}\left(\psi_{i}(\zeta)\right)^{l-k} \gamma_{i, k}(\zeta)}_{=: Q_{l}(\zeta)} .
$$

Furthermore, using the formal power series expansion of the exponential function, the operational function $\hat{C}$ can be written as

$$
\hat{C}(x)=\sum_{i=1}^{n} \sum_{k=0}^{\kappa_{i}-1} \sum_{l=k}^{\infty} s^{l-\nu} \frac{x^{l}}{(l-k) !} \gamma_{i, k}\left(s^{-\sigma}\right)\left(\psi_{i}\left(s^{-\sigma}\right)\right)^{l-k}
$$

Rearranging the sums we obtain

$$
\hat{C}(x)=\sum_{l=0}^{\infty} x^{l} s^{l-\nu} \underbrace{\sum_{i=1}^{n} \sum_{k=0}^{\min \left(\kappa_{i}-1, l\right)} \frac{1}{(l-k) !} \gamma_{i, k}\left(s^{-\sigma}\right)\left(\psi_{i}\left(s^{-\sigma}\right)\right)^{l-k}}_{=Q_{l}\left(s^{-\sigma}\right)} .
$$

Due to the assumption, $s^{l-\nu} Q_{l}\left(s^{-\sigma}\right)$ is a polynomial in $s$ for $l \in \mathbb{N}$. Furthermore, introducing

$$
q_{l, i, k}(\zeta)=\frac{\gamma_{i, k}(\zeta)(\psi(\zeta))^{l-k}}{(l-k) !}, \quad l \geq k,
$$

the sums $Q_{l}$ can be rewritten as

$$
Q_{l}(\zeta)=\sum_{i=1}^{n} \sum_{k=0}^{\min \left(l, \kappa_{i}-1\right)} q_{l, i, k}(\zeta), \quad l \in \mathbb{N} .
$$

Since, by assumption, the $\psi_{i}, \gamma_{i, k}$ are analytic in 0 , the functions $q_{l, i, k}$ are analytic in 0 for $k \leq l$. Thus, according to Lemma 2.5, the $Q_{l}$ are polynomials satisfying $\sigma \operatorname{deg} Q_{l}(\zeta) \leq l-\nu$. Therefore, it follows from equation (2.10) that the $X_{m}$ are polynomials in $\zeta$ as well, where $\sigma \operatorname{deg} X_{m}(\zeta ; x, t) \leq \sigma m-1$, which implies $X_{m}^{(m)}(\zeta ; x, t)=0$.

\section{Distributed Delay interpretation of the motion Planning EQUATions}

In this section we will prove that $\mathcal{W}_{i, j}$ and $\mathcal{V}_{j}$, as defined in Section 1, are finite distributed delay operators. To this end, we show that they have compact support with respect to $t$ and they can be written as sums of functions $\mathbb{R} \rightarrow \mathbb{C}$ and Dirac distributions. The set of compact support functions and Dirac distributions forms a subring of $\mathcal{M}$. It is, therefore, sufficient to show the following:

Theorem 3.1. The elements of $\mathfrak{C}^{*}$ and, therefore, also those of $\mathfrak{D}^{*}$ have compact support (w.r.t. time) and contain only Dirac distributions of first order (the unit of $\mathcal{M}$ and its translations by shifts).

Proof. As for the definition of the operational functions in $\mathfrak{C}$ (see Sect. 1), we can write the operational functions in $\mathfrak{C}^{*}$ as $\hat{C}_{i, j, \mu}^{*}(x)=\sum_{k \in I_{i}} \sum_{l=0}^{\kappa_{k}-i} x^{l} \hat{F}_{i, j, k, l, \mu}^{*}(x)$ with $\hat{F}_{i, j, k, l, \mu}^{*}(x)=s^{\mu} \eta_{i, j, k, l} \exp \left(x \lambda_{k}\right), i=1, \ldots, \kappa_{\max }, j=$ $1, \ldots, n_{i}, k \in I_{i}, l=0, \ldots, \kappa_{k}-i, \mu=0, \ldots, N_{i}-\kappa_{\max }+i-j$. Using (2.1) we can rewrite the functions $F_{i, j, k, l, \mu}^{*}$ as

$$
\hat{F}_{i, j, k, l, \mu}^{*}(x)=s^{-N_{i}+j+l+\mu} \tilde{\eta}_{i, j, k, l} \exp \left(s x \tilde{\lambda}_{k}\right) .
$$


Due to Lemmas 2.1 and 2.2 the assumptions of Theorem 2.4 are satisfied. It follows that the functions $F_{i, j, k, l, \mu}^{*}$ with $\mu<N_{i}-\kappa_{i}+i+j$ yield piecewise continuous functions, whereas those with $\mu=N_{i}-\kappa_{i}+i+j$ contain first order Dirac distributions in addition.

According to Lemma 2.3 the operational functions $\mathfrak{C}^{*}$ can be written as formal power series with coefficients in $\mathbb{C}[s]$. Thus, by Theorem 2.6 it follows that the functions (of $t$ ) obtained by interpreting the operational functions $\mathfrak{C}^{*}$ have compact support with respect to $t$.

Remark 3.2. We have shown that the convolution kernels $\mathcal{V}_{j}$ and $W_{i, j}$ in (1.9) have compact support and can be evaluated by convolving (compact support) functions $C_{i, j}^{*}$, which can be written using infinite series. Alternatively, the convolution kernels in (1.9) may be interpreted by substituting the operational functions in $\mathfrak{C}^{*}$ and $\mathfrak{D}^{*}$ by their definitions, i.e., by sums of exponential (operational) functions. Expanding the products of these sums, we obtain a sum of functions of the form (2.3), which may be verified to satisfy the assumptions of Theorem 2.4. Hence, the convolution kernels in (1.9) can be written as infinite series and without using convolution integrals. We only need the Taylor coefficients of the series expansion of the roots of the characteristic equation, which can be calculated recursively from the characteristic equation. Hence, the method is suitable even for higher order problems.

Remark 3.3. When identifying the operational form of the integral kernels (1.9) with a Fourier transform, their compact support could be verified by use of the Paley-Wiener theorem as well (see [19]). Since the PaleyWiener theorem is an existence theorem, contrary to our approach, it does not yield explicit formulae for the convolution kernels that we need for motion planning.

\section{Applichtion to a Timoshenko Beam model}

\subsection{Mathematical model}

We consider a Timoshenko beam mounted radially on a rigid hub (cf. Fig. 1). A punctual load $\tilde{m}$ is fixed at its free end ${ }^{6}$. The position of the beam is controlled via the rotation angle $\tilde{\theta}$ of the hub. Its motion is governed by the following system of partial differential equations ${ }^{7}$ :

$$
\begin{aligned}
(\tilde{x}+\tilde{r}) A \varrho \tilde{\theta}_{\tilde{t} \tilde{t}}(\tilde{t}) & =A \varrho \tilde{w}_{\tilde{t} \tilde{t}}(\tilde{x}, \tilde{t})-k G A\left(\tilde{\varphi}_{\tilde{x}}(\tilde{x}, \tilde{t})+\tilde{w}_{\tilde{x} \tilde{x}}(\tilde{x}, \tilde{t})\right) \\
-I \varrho \tilde{\theta}_{\tilde{t} \tilde{t}}(\tilde{t}) & =I \varrho \tilde{\varphi}_{\tilde{t} \tilde{t}}(\tilde{x}, \tilde{t})-E I \tilde{\varphi}_{\tilde{x} \tilde{x}}(\tilde{x}, \tilde{t})+k G A\left(\tilde{\varphi}(\tilde{x}, \tilde{t})+\tilde{w}_{\tilde{x}}(\tilde{x}, \tilde{t})\right)
\end{aligned}(\tilde{x}, \tilde{t}) \in[0, \tilde{l}] \times \mathbb{R} .
$$

Here $\tilde{w}$ denotes the displacement of the centerline and $\tilde{\varphi}$ the rotation angle of the cross sections. Cross section area $A$, mass density $\varrho$, moment of inertia $I$, Young's modulus $E$, and shear modulus $G$ are constant parameters. Moreover, $k$ denotes a parameter that depends on the shape of the cross section. The boundary conditions are given by

$$
\tilde{\varphi}(0, \tilde{t})=\tilde{\theta}(\tilde{t}), \quad \tilde{w}(0, \tilde{t})=-\tilde{r} \tilde{\theta}(\tilde{t}), \quad \tilde{\varphi}_{\tilde{x}}(\tilde{l}, \tilde{t})=0, \quad k G A\left(\tilde{w}_{\tilde{x}}(\tilde{l}, \tilde{t})+\tilde{\varphi}(\tilde{l}, \tilde{t})\right)+\tilde{m} \tilde{w}_{\tilde{t} \tilde{t}}(\tilde{x}, \tilde{t})=0
$$

where $\tilde{r}$ is the radius of the hub, $\tilde{m}$ is the mass of the load, and $\tilde{l}$ the length of the beam. The initial conditions are assumed to be zero:

We introduce the variables

$$
\tilde{w}(\tilde{x}, 0)=\tilde{w}_{\tilde{t}}(\tilde{x}, 0)=\tilde{\varphi}(\tilde{x}, 0)=\tilde{\varphi}_{\tilde{t}}(\tilde{x}, 0)=0 .
$$

$$
t=\frac{1}{\tilde{l}^{2}} \sqrt{\frac{E I}{A \varrho}} \tilde{t}, \quad x=1-\frac{\tilde{x}}{\tilde{l}}, \quad \theta(t)=\tilde{l} \tilde{\theta}(\tilde{t}), \quad w(x, t)=\tilde{w}(\tilde{x}, \tilde{t})-(\tilde{x}+\tilde{r}) \tilde{\theta}(\tilde{t}), \quad \varphi(x, t)=\tilde{l}(\tilde{\varphi}(\tilde{x}, \tilde{t})+\tilde{\theta}(\tilde{t}))
$$

\footnotetext{
${ }^{6}$ A clamped Timoshenko beam driven by a boundary torque acting on the free end has been considered in [21].

${ }^{7}$ The model can be derived by linearizing the geometrically exact models given in [24] and [22].
} 


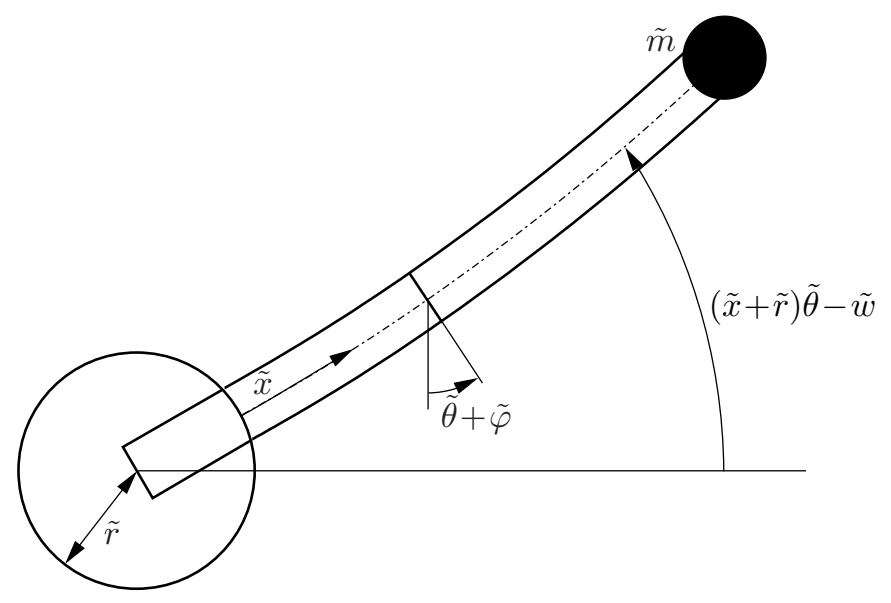

FIGURE 1. Timoshenko beam moving in a plane.

and the constant parameters

$$
\mu_{1}=\frac{1}{\tilde{l}^{2}} \frac{I}{A}, \quad \quad \mu_{2}=\frac{1}{\tilde{l}^{2}} \frac{I}{A} \frac{E}{k G}, \quad m=\frac{1}{\tilde{l} A \varrho} \tilde{m}, \quad r=\frac{\tilde{r}}{\tilde{l}},
$$

where we assume $\mu_{1}, \mu_{2} \in \mathbb{R}^{+}$and $\mu_{2} \neq \mu_{1}$. From (4.1) we obtain the transformed equations on $[0,1] \times \mathbb{R}^{+} \ni(x, t)$

$$
\begin{aligned}
& 0=\mu_{2} \ddot{w}(x, t)+\left(\varphi^{\prime}(x, t)-w^{\prime \prime}(x, t)\right) \\
& 0=\mu_{1} \mu_{2} \ddot{\varphi}(x, t)-\mu_{2} \varphi^{\prime \prime}(x, t)+\left(\varphi(x, t)-w^{\prime}(x, t)\right),
\end{aligned}
$$

and from (4.2) the transformed boundary conditions

$$
\varphi(1, t)=\theta(t), \quad w(1, t)=-r \theta(t), \quad \varphi^{\prime}(0, t)=0, \quad \varphi(0, t)-w^{\prime}(0, t)+m \mu_{2} \ddot{w}(0, t)=0 .
$$

A (normalized) Euler-Bernoulli beam model can be obtained from (4.3) using the Euler-Bernoulli assumption $\varphi=w^{\prime}$, additionaly, neglecting the rotational intertia $\left(\mu_{1}=0\right)$. Flatness based motion planning for the Euler-Bernoulli beam model which does not belong to the class of systems considered in this paper has been investigated in $[6]^{8}$.

${ }^{8}$ Substituting (4.3a) into the derivative w.r.t. $x$ of (4.3b) we obtain a modified version of (4.3):

$$
0=\mu_{2} \ddot{w}(x, t)+\left(\varphi^{\prime}(x, t)-w^{\prime \prime}(x, t)\right), \quad 0=\mu_{1} \ddot{\varphi}^{\prime}(x, t)-\varphi^{\prime \prime \prime}(x, t)-\ddot{w}(x, t) .
$$

With $\varphi=w^{\prime}$, from the first equation we obtain $\mu_{2}=0$. Hence, using $\mu_{1}=0$ and eliminating $\phi$ from the second equation, we obtain $w^{I V}(x, t)+\ddot{w}(x, t)=0$. Since the characteristic equation $\lambda^{4}+s^{2}=0$ does not satisfy Assumption $1.2^{*}$ here, we do not obtain a solution involving distributed delays. Instead, this equation leads to series representations involving flat output derivatives of arbitrary order [6].

For small values of $\mu_{1}$ and $\mu_{2}$ the Timoshenko model may be understood as perturbation of the Euler-Bernoulli model. The link between the completely different representations of the solutions is currently investigated, i.e. we consider a Timoshenko beam model and investigate the limit $\mu_{1}, \mu_{2} \rightarrow 0$. 


\subsection{Operational solution}

As described in the first section, we interprete the function $\theta$ as an operator $\hat{\theta} \in \mathcal{M}$ and the functions $\varphi, w$ as operational functions $\hat{\varphi}, \hat{w}:[0,1] \rightarrow \mathcal{M}$. From (4.3) we obtain the ordinary differential equations

$$
\begin{aligned}
& 0=\mu_{2} s^{2} \hat{w}(x)+\left(\hat{\varphi}^{\prime}(x)-\hat{w}^{\prime \prime}(x)\right) \\
& 0=\mu_{1} \mu_{2} s^{2} \hat{\varphi}(x)-\mu_{2} \hat{\varphi}^{\prime \prime}(x)+\left(\hat{\varphi}(x)-\hat{w}^{\prime}(x)\right) .
\end{aligned}
$$

The boundary conditions (4.4) can be rewritten as

$$
\begin{aligned}
\hat{\varphi}(1) & =\hat{\theta}, & \hat{w}(1) & =-r \hat{\theta} \\
\hat{\varphi}^{\prime}(0) & =0, & \hat{\varphi}(0)-\hat{w}^{\prime}(0)+m \mu_{2} s^{2} \hat{w}(0) & =0 .
\end{aligned}
$$

The characteristic equation of $(4.5)$

$$
\lambda^{4}-s^{2}\left(\mu_{1}+\mu_{2}\right) \lambda^{2}+s^{2}\left(1+s^{2} \mu_{1} \mu_{2}\right)=0
$$

satisfies the Assumption 1.1*. It has the roots $\lambda_{i}=s \psi_{i}\left(s^{-2}\right), i=1, \ldots, 4$ with multiplicities $\kappa_{i}=1$, where

$$
\psi_{1}(\zeta)=-\psi_{3}(\zeta)=\sqrt{\frac{\mu_{2}+\mu_{1}}{2}+\sqrt{\frac{\left(\mu_{1}-\mu_{2}\right)^{2}}{4}-\zeta}}, \quad \psi_{2}(\zeta)=-\psi_{4}(\zeta)=\sqrt{\frac{\mu_{2}+\mu_{1}}{2}-\sqrt{\frac{\left(\mu_{1}-\mu_{2}\right)^{2}}{4}-\zeta}}
$$

Obviously, Assumption $1.2^{*}$ is satisfied for positive $\mu_{2}$ and $\mu_{1}$ with $^{9} \mu_{2} \neq \mu_{1}$. According to equation ${ }^{10,11}$ (1.6a) we define the operational functions in $\mathfrak{C}$ as $(j=1, \ldots, 4)$

$$
\hat{C}_{j}(x)=\sum_{k=1}^{4} \hat{F}_{j, k}(x), \quad \hat{F}_{j, k}(x)=\eta_{j, k} \mathrm{e}^{x \lambda_{j}}, \quad \eta_{j, k}=s^{-4+j} \gamma_{j, k}\left(s^{-2}\right), \quad \gamma_{j, k}(\zeta)=\frac{\left(\psi_{k}(\zeta)\right)^{j-1}}{\prod_{l=1, l \neq k}^{4}\left(\psi_{k}(\zeta)-\psi_{l}(\zeta)\right)}
$$

Using these operational functions, we can write the following system of fundamental solutions:

$$
\begin{array}{ll}
\hat{\mathbf{W}}_{1}(x)=\left(\begin{array}{c}
\left(1+s^{2} \mu_{2} \mu_{1}\right) \hat{C}_{1}(x) \\
\left(1-s^{2} \mu_{2}^{2}\right) \hat{C}_{2}(x)+\mu_{2} \hat{C}_{4}(x)
\end{array}\right), & \hat{\mathbf{W}}_{2}(x)=\left(\begin{array}{c}
\hat{C}_{2}(x) \\
-s^{2} \mu_{2} \hat{C}_{1}(x)+\hat{C}_{3}(x)
\end{array}\right), \\
\hat{\mathbf{W}}_{3}(x)=\left(\begin{array}{c}
\hat{C}_{3}(x) \\
-s^{2} \mu_{2} \hat{C}_{2}(x)+\hat{C}_{4}(x)
\end{array}\right), & \hat{\mathbf{W}}_{4}(x)=\left(\begin{array}{c}
\hat{C}_{4}(x) \\
-s^{2}\left(1+s^{2} \mu_{1} \mu_{2}\right) \hat{C}_{1}(x)+s^{2} \mu_{1} \hat{C}_{3}(x)
\end{array}\right) .
\end{array}
$$

\footnotetext{
${ }^{9}$ The physically irrelevant case where the characteristics of the PDEs (4.3) coincide is characterized by $\mu:=\mu_{2}=\mu_{1}$. Though the characteristic equation does not satisfy Assumption $1.2 *$, the method could be applied in a slightly modified way. This is due to the fact, that the characteristic polynomial can be written as $P(\lambda)=\left(\lambda^{2}-s^{2} \mu+\mathrm{i} s\right)\left(\lambda^{2}-s^{2} \mu-\mathrm{i} s\right)$, where, obviously, both parts of $P$ satisfy Assumption $1.2^{*}$. Therefore, we obtain linear combinations of $\exp \left( \pm x s \sqrt{\mu \pm \text { is } s^{-1}}\right)$ which can be identified with Bessel functions, $c f .[2,20]$.

${ }^{10}$ Since in this application we have no multiple roots, we omit the related indices, which are meaningless in this case, for example we write $\hat{C}_{j}$ instead of $\hat{C}_{1, j}$ and $\eta_{j, k}$ instead of $\eta_{1, j, k, 0}$.

${ }^{11}$ Alternatively, the functions in $\mathfrak{C}$ can be written, using a notation, similar tho that given in [21]

$$
\begin{array}{ll}
\hat{C}_{1}(x)=\frac{1}{\lambda_{1}^{2}-\lambda_{2}^{2}}\left(\frac{\sinh \left(x \lambda_{1}\right)}{\lambda_{1}}-\frac{\sinh \left(x \lambda_{2}\right)}{\lambda_{2}}\right), & \hat{C}_{2}(x)=\frac{1}{\lambda_{1}^{2}-\lambda_{2}^{2}}\left(\cosh \left(x \lambda_{1}\right)-\cosh \left(x \lambda_{2}\right)\right) \\
\hat{C}_{3}(x)=\frac{1}{\lambda_{1}^{2}-\lambda_{2}^{2}}\left(\lambda_{1} \sinh \left(x \lambda_{1}\right)-\lambda_{2} \sinh \left(x \lambda_{2}\right)\right), & \hat{C}_{4}(x)=\frac{1}{\lambda_{1}^{2}-\lambda_{2}^{2}}\left(\lambda_{1}^{2} \cosh \left(x \lambda_{1}\right)-\lambda_{2}^{2} \cosh \left(x \lambda_{2}\right)\right) .
\end{array}
$$
}


With the Ansatz

$$
\left(\begin{array}{l}
\hat{w}(x) \\
\hat{\varphi}(x)
\end{array}\right)=\sum_{i=1}^{4} \hat{K}_{i} \hat{\mathbf{W}}_{i}(x)
$$

the boundary conditions (4.6b) yield

$$
\hat{K}_{1}=-m s^{2} \hat{K}_{4}, \quad \hat{K}_{2}=-\mu_{1} s^{2} \hat{K}_{4}
$$

Together with (4.6a) it follows

$$
\begin{aligned}
0= & \hat{K}_{4}[\underbrace{r m s^{2}\left(\left(\mu_{2}^{2} s^{2}-1\right) \hat{C}_{2}(1)-\mu_{2} \hat{C}_{4}(1)\right)-\mu_{1} s^{2} \hat{C}_{2}(1)+\hat{C}_{4}(1)-m s^{2}\left(1+s^{2} \mu_{1} \mu_{2}\right) \hat{C}_{1}(1)-r s^{2} \hat{C}_{1}(1)}_{\hat{k}_{4}}] \\
& +\hat{K}_{3}[\underbrace{\hat{C}_{2}(1)+\hat{C}_{4}(1)-r \mu_{2} s^{2} \hat{C}_{3}(1)}_{\hat{k}_{3}}] \\
\hat{\theta} & =\left(-\mu_{2} s^{2} \hat{C}_{2}(1)+\hat{C}_{4}(1)\right) \hat{K}_{3}+s^{2}\left(m\left(\mu_{2}^{2} s^{2}-1\right) \hat{C}_{2}(1)-m \mu_{2} \hat{C}_{4}(1)-\hat{C}_{1}(1)\right) \hat{K}_{4} .
\end{aligned}
$$

Equation (4.10a) is satisfied if we introduce the flat output $\hat{y}$ by $\hat{K}_{3}=\hat{k}_{4} \hat{y}, \hat{K}_{4}=-\hat{k}_{3} \hat{y}$. The remaining coefficients and the angle $\hat{\theta}$ can be calculated by substituting $\hat{K}_{1}$ and $\hat{K}_{5}$ in (4.9) and (4.10b), respectively. Finally, after substituting $s^{\mu} C_{j}(j+\mu \leq 4)$ by $C_{j, \mu}^{*}$ as described in Section 1 , we obtain

$$
\hat{\varphi}(x)=\hat{\Phi}_{0}(x) \hat{y}+\hat{\Phi}_{1}(x) s \hat{y}, \quad \hat{w}(x)=\hat{W}_{0}(x) \hat{y}+\hat{W}_{1}(x) s \hat{y}, \quad \hat{\theta}=\hat{\Theta}_{0} \hat{y}+\hat{\Theta}_{1} \hat{y}
$$

with

$$
\begin{aligned}
& \hat{W}_{0}(x)=\left(\begin{array}{c}
\mu_{1}\left(\hat{C}_{2,2}^{*}(x) \hat{C}_{3,0}^{*}(1)-\hat{C}_{3,0}^{*}(x) \hat{C}_{2,2}^{*}(1)\right)+\hat{C}_{3,0}^{*}(x) \hat{C}_{4,0}^{*}(1)-\hat{C}_{3,0}^{*}(1) \hat{C}_{4,0}^{*}(x) \\
\left(\hat{C}_{4,0}^{*}(x)-\mu_{1} \hat{C}_{2,2}^{*}(x)\right)\left(\mu_{2} \hat{C}_{2,2}^{*}(1)-\hat{C}_{4,0}^{*}(1)\right)-\hat{C}_{1,2}^{*}(1) \hat{C}_{3,0}^{*}(x) \\
\hat{C}_{1,2}^{*}(x) \hat{C}_{3,0}^{*}(1)-\hat{C}_{1,2}^{*}(1) \hat{C}_{3,0}^{*}(x)+\mu_{2} \mu_{1}\left(\hat{C}_{1,3}^{*}(x) \hat{C}_{3,1}^{*}(1)-\hat{C}_{1,3}^{*}(1) \hat{C}_{3,1}^{*}(x)\right)
\end{array}\right)^{T}\left(\begin{array}{c}
1 \\
r \\
m
\end{array}\right) \\
& \hat{W}_{1}(x)=\left(\left(\mu_{2} \hat{C}_{3,1}^{*}(x)-\hat{C}_{1,1}^{*}(x)-\mu_{2} \mu_{1} \hat{C}_{1,3}^{*}(x)\right)\left(\mu_{2} \hat{C}_{2,2}^{*}(1)-\hat{C}_{4,0}^{*}(1)\right)-\hat{C}_{2,1}^{*}(1) \hat{C}_{3,0}^{*}(x)\right) r m \\
& \hat{\Phi}_{0}(x)=\left(\begin{array}{c}
\hat{C}_{1,2}^{*}(x) \hat{C}_{3,0}^{*}(1)+\left(\mu_{2} \hat{C}_{2,2}^{*}(x)-\hat{C}_{4,0}^{*}(x)\right)\left(\mu_{1} \hat{C}_{2,2}^{*}(1)-\hat{C}_{4,0}^{*}(1)\right) \\
\mu_{2}\left(\hat{C}_{1,2}^{*}(1) \hat{C}_{2,2}^{*}(x)-\hat{C}_{1,2}^{*}(x) \hat{C}_{2,2}^{*}(1)\right)+\left(\hat{C}_{1,2}^{*}(x) \hat{C}_{4,0}^{*}(1)-\hat{C}_{1,2}^{*}(1) \hat{C}_{4,0}^{*}(x)\right) \\
\left(\hat{C}_{2,2}^{*}(x) \hat{C}_{4,0}^{*}(1)-\hat{C}_{2,2}^{*}(1) \hat{C}_{4,0}^{*}(x)\right)
\end{array}\right)\left(\begin{array}{c}
1 \\
r \\
r m
\end{array}\right) \\
& \hat{\Phi}_{1}(x)=\left(\hat{C}_{2,1}^{*}(x) \hat{C}_{3,0}^{*}(1)+\left(\hat{C}_{2,2}^{*}(x) \mu_{2}-\hat{C}_{4,0}^{*}(x)\right)\left(\hat{C}_{1,1}^{*}(1)+\mu_{2} \mu_{1} \hat{C}_{1,3}^{*}(1)-\mu_{2} \hat{C}_{3,1}^{*}(1)\right)\right) m \\
& \hat{\Theta}_{0}=\hat{C}_{1,2}^{*}(1) \hat{C}_{3,0}^{*}(1)+\left(\mu_{2} \hat{C}_{2,2}^{*}(1)-\hat{C}_{4,0}^{*}(1)\right)\left(\mu_{1} \hat{C}_{2,2}^{*}(1)-\hat{C}_{4,0}^{*}(1)\right) \\
& \hat{\Theta}_{1}=\left(\hat{C}_{2,1}^{*}(1) \hat{C}_{3,0}^{*}(1)+\left(\hat{C}_{2,2}^{*}(1) \mu_{2}-\hat{C}_{4,0}^{*}(1)\right)\left(\hat{C}_{1,1}^{*}(1)+\mu_{2} \mu_{1} \hat{C}_{1,3}^{*}(1)-\mu_{2} \hat{C}_{3,1}^{*}(1)\right)\right) m .
\end{aligned}
$$




\subsection{Motion planning}

Since $\mu_{1}, \mu_{2} \in \mathbb{R}^{+}$and $\mu_{1} \neq \mu_{2}$, it follows $\psi_{i}(0) \in \mathbb{R}$ and $\psi_{i}(0) \neq \psi_{j}(0)$ for $j \neq i$. Hence, we can use Theorem 2.4 with $\eta=\eta_{j, k}$ and $\lambda=\lambda_{k}$ to interprete the operational functions $\hat{F}_{j, k}$ in (4.7). By Theorem 2.6 the operators defined in (4.7) have support in $I \subseteq\left\{(x, t) \mid x \in \mathbb{R}, t \in\left[-|x| \max \left(\mu_{1}, \mu_{2}\right),|x| \max \left(\mu_{1}, \mu_{2}\right)\right]\right\}$.

Writing the (convolution) products in (4.11) as integrals yields

$$
\begin{aligned}
\varphi(x, t) & =\int_{I(x)}\left(\Phi_{0}(x, \tau) y(t-\tau)+\Phi_{1}(x, \tau) \dot{y}(t-\tau)\right) \mathrm{d} \tau \\
w(x, t) & =\int_{I(x)}\left(W_{0}(x, \tau) y(t-\tau)+W_{1}(x, \tau) \dot{y}(t-\tau)\right) \mathrm{d} \tau \\
\theta(t) & =\int_{I(1)}\left(\Theta_{0}(\tau) y(t-\tau)+\Theta_{1}(\tau) \dot{y}(t-\tau)\right) \mathrm{d} \tau
\end{aligned}
$$

with $I(x)=\left[-\max \left(\mu_{2}, \mu_{1}\right)(1+x), \max \left(\mu_{2}, \mu_{1}\right)(1+x)\right]$.

Now a transition from rest to rest satisfying the zero initial conditions can be parameterized by choosing a $C^{1}$-function as the trajectory $t \mapsto y(t)$, with $y(t)=0 \forall t<2 \max \left(\mu_{2}, \mu_{1}\right)$ and $y(t)=y^{*} \forall t \geq t^{*}+2 \max \left(\mu_{2}, \mu_{1}\right)$, with $t^{*} \in \mathbb{R}^{+}, y^{*} \in \mathbb{R}$. The trajectory for the system variables can be calculated from the flat output trajectory by evaluating the convolution integrals (4.12).

\section{A. Background From Mikusiński's operational CALCUlus}

The set of continuous functions $[0,+\infty) \rightarrow \mathbb{C}$ equipped with the sum,$+(f+g)(t)=f(t)+g(t)$, and the convolution product $\star,(f \star g)(t)=(g \star f)(t)=\int_{0}^{t} f(\tau) g(t-\tau) \mathrm{d} \tau=\int_{0}^{t} g(\tau) f(t-\tau) \mathrm{d} \tau$ is a commutative $\operatorname{ring} \mathcal{C}$. According to Titchmarsh's theorem (see, e.g. $[12,13,23]), \mathcal{C}$ is integral, i.e., it has no zero divisors:

$$
f \star g=0 \quad \Leftrightarrow \quad f=0 \text { or } g=0 .
$$

The quotient field $\mathcal{M}$ of $\mathcal{C}$ is called the Mikusiński field. The elements of $\mathcal{M}$ are called operators. The (convolution) product of two operators $a, b \in \mathcal{M}$ is also written $a b$ (instead of $a \star b$ ). If a function $f \in \mathcal{C}$ is considered as an operator in $\mathcal{M}$ the notation $\{f\}$ is used ${ }^{12}$.

Examples: The Dirac operator is the neutral element 1 in $\mathcal{M}$. It is the analogue in Mikusiński's approach of the Dirac measure $\delta$ in Schwartz distribution theory. The Dirac operator 1 should not be confused with the Heaviside function. The inverse in $\mathcal{M}$ of the Heaviside function is the differential operator $s$. If $f \in \mathcal{C}$ is $C^{1}$, then $\{\dot{f}\}=s\{f\}-f(0)$, where $\dot{f}$ is the derivative of $f$. Operators from the subfield $\mathbb{C}(s)$ of rational functions in the indeterminate $s$ with complex coefficients possess a meaning analogue to that in the Laplace calculus. For instance,

$$
\frac{1}{(s-a)^{n}}=\left\{\frac{t^{n-1} \exp (a t)}{(n-1) !}\right\}, \quad 0<n \in \mathbb{N} .
$$

The exponential function $\mathrm{e}^{s \alpha}(\alpha \in \mathbb{R})$ is the shift operator: $\mathrm{e}^{s \alpha} \hat{f}=\{f(t+\alpha)\}$.

A sequence $a_{n}, n \geq 0$ of operators is said to be operationally convergent [12,13] if there exists an operator $p$ such that the products $a_{n} p$ belong to $\mathcal{C}$ and converge in $\mathcal{C}$ almost uniformly, i.e., uniformly on any compact, to an element of $\mathcal{C}$. A series $\sum_{\nu \geq 0} b_{\nu}$ of operators is said to be operationally convergent if the sequence $\sum_{\nu=0}^{n} b_{\nu}$ has this property. An operational function is a mapping $I \rightarrow \mathcal{M}, I \subseteq \mathbb{R}$. Its continuity, differentiability and integrability are defined in $[12,13]$.

\footnotetext{
${ }^{12}$ In this paper we use the hat to distinguish the functional notation from the operational one: $\hat{f}=\{f(t)\}$.
} 
Lemma A.1 (see [12], p. 179). If the series

$$
\sum_{k=0}^{\infty} a_{k} \lambda^{k}, \quad \lambda \in \mathbb{C}, a_{k} \in \mathbb{C}
$$

has a positive radius of convergence then the series $\sum_{k=0}^{\infty} a_{k} \hat{f}^{k}, \hat{f} \in \mathcal{C}$ is operationally convergent.

Lemma A.2. Define $\mathcal{C}^{*}$ as the subring of $\mathcal{M}$ generated by complex multiples of the unit in $\mathcal{M}$ over $\mathcal{C}\left(\mathcal{C}^{*}=\right.$ $\mathcal{C} \cup \mathbb{C})$. If the series (A.2) has an infinite radius of convergence then the series

$$
\sum_{k=0}^{\infty} a_{k} \hat{g}^{k}, \quad \hat{g} \in \mathcal{C}^{*}
$$

is operationally convergent.

Proof. Rewriting the operator $\hat{g}$ in the form $\hat{g}=\hat{f}+\alpha, \alpha \in \mathbb{C}, f \in \mathcal{C}$ we obtain from (A.3)

$$
\sum_{k=0}^{\infty} a_{k} \hat{g}^{k}=s \sum_{k=0}^{\infty} a_{k} \frac{1}{s}(\hat{f}+\alpha)^{k}=s\left\{\sum_{k=0}^{\infty} a_{k} g_{k}(t)\right\}
$$

where $g_{0}(t)=h(t)$ and $g_{k+1}(t)=\int_{0}^{t} g_{k}(\tau) f(t-\tau) \mathrm{d} \tau+\alpha g_{k}(\tau)$. For every $t_{0}>0$ there exists an $M \geq|\alpha|$ such that $M>|f(t)| \forall t \in\left[0, t_{0}\right]$. We show by induction that

$$
\left|g_{k}(t)\right| \leq M^{k} \sum_{n=0}^{k}\left(\begin{array}{l}
k \\
n
\end{array}\right) \frac{t^{n}}{n !}, \quad t \in\left[0, t_{0}\right]
$$

Assuming that the inequality (A.4) holds for some $k=k_{0} \in \mathbb{N}$ it follows

$$
\begin{aligned}
g_{k_{0}+1}(t) & =\int_{0}^{t} g_{k_{0}}(\tau) f(t-\tau) \mathrm{d} \tau+\alpha g_{k_{0}}(\tau) \leq \int_{0}^{t}\left(M^{k_{0}+1} \sum_{n=0}^{k_{0}}\left(\begin{array}{c}
k_{0} \\
n
\end{array}\right) \frac{\tau^{n}}{n !}\right) \mathrm{d} \tau+M^{k_{0}+1} \sum_{n=0}^{k_{0}}\left(\begin{array}{c}
k_{0} \\
n
\end{array}\right) \frac{t^{n}}{n !} \\
& =M^{k_{0}+1}\left(\sum_{n=1}^{k_{0}+1}\left(\begin{array}{c}
k_{0} \\
n-1
\end{array}\right) \frac{t^{n}}{n !}+\sum_{n=0}^{k_{0}}\left(\begin{array}{c}
k_{0} \\
n
\end{array}\right) \frac{t^{n}}{n !}\right)=M^{k_{0}+1} \sum_{n=0}^{k_{0}+1}\left(\begin{array}{c}
k_{0}+1 \\
n
\end{array}\right) \frac{t^{n}}{n !}, \quad t \in\left[0, t_{0}\right] .
\end{aligned}
$$

Hence, inequality (A.4) holds for $k=k_{0}+1$ and

$$
\left|g_{k}(t)\right| \leq M^{k} \sum_{n=0}^{k}\left(\begin{array}{l}
k \\
n
\end{array}\right) \frac{t^{n}}{n !} \leq M^{k}(t+1)^{k}, \quad t \in\left[0, t_{0}\right]
$$

Due to the assumption, the series $\sum_{k=0}^{\infty} a_{k}(M(t+1))^{k}$ is convergent. It follows the uniform convergence of $\sum_{k=0}^{\infty} a_{k} g_{k}$ and, therefore, the operational convergence of (A.3).

Lemma A.3. Consider the ordinary initial value problem

$$
\sum_{i=0}^{N} p_{i} f^{(i)}(x)=0, \quad f^{(j)}(0)=0, \quad j=0, \ldots, N-2, \quad f^{(N-1)}(0)=1, \quad p_{i} \in K
$$


where $K$ is an algebraically closed field. Then, the $k$-th derivative of the solution $f$ is given by

$$
f^{(k)}(x)=\sum_{i=1}^{n} \sum_{j=0}^{\kappa_{i}-1} \frac{x^{j} \exp \left(x s_{i}\right)}{j !\left(\kappa_{i}-j-1\right) !} \frac{\partial^{\kappa_{i}-j-1}}{\partial s_{i}^{\kappa_{i}-j-1}}\left(\frac{s_{i}^{k}}{\prod_{l=1, l \neq i}^{n}\left(s_{i}-s_{l}\right)^{\kappa_{l}}}\right) .
$$

Proof. As usual we replace $f^{(i)}$ by $s^{i} \hat{f}-\sum_{j=0}^{N-1} s^{N-j-1} f^{(j)}(0)$ and obtain the operational solution

$$
\left\{f^{(k)}(x)\right\}=\frac{s^{k}}{P(s)} \quad \text { with } \quad P(s)=\prod_{i=1}^{n}\left(s-s_{i}\right)^{\kappa_{i}} .
$$

Here $P(s)$ is the characteristic polynomial of (A.6) with roots $s_{i}(i=1, \ldots, n)$ in $K$, of multiplicity $\kappa_{i}$. The solution is expanded into partial fractions. To this end, we determine coefficients $a_{i, j, k} \in K,(i=1, \ldots, n$, $\left.j=1, \ldots, \kappa_{i}, k=0, \ldots, n-1\right)$ such that the equation

$$
\frac{s^{k}}{\prod_{l=1}^{n}\left(s-s_{l}\right)^{\kappa_{l}}}=\sum_{i=1}^{n} \sum_{j=1}^{\kappa_{i}} \frac{a_{i, j, k}}{\left(s-s_{i}\right)^{j}}
$$

is satisfied.

Multiplying (A.7) by $\left(s-s_{\mu}\right)^{\kappa_{\mu}}, \mu=1, \ldots, n$ yields

$$
\frac{s^{k}}{\prod_{l=1, l \neq \mu}^{n}\left(s-s_{l}\right)^{\kappa_{l}}}=\sum_{j=1}^{\kappa_{\mu}} a_{\mu, j, k}\left(s-s_{\mu}\right)^{\kappa_{\mu}-j}+\sum_{i=1, i \neq \mu}^{n} \sum_{j=1}^{\kappa_{i}} \frac{a_{i, j, k}\left(s-s_{\mu}\right)^{\kappa_{\mu}}}{\left(s-s_{i}\right)^{j}} .
$$

For $1 \leq \nu \leq \kappa_{\mu}$ we differentiate $\kappa_{\mu}-\nu$ times with respect to $s$, evaluate at $s=s_{\mu}$, and obtain

$$
a_{\mu, \nu, k}=\frac{1}{\left(\kappa_{\mu}-\nu\right) !} \frac{\partial^{\kappa_{\mu}-\nu}}{\partial s_{\mu}^{\kappa_{\mu}-\nu}}\left(\frac{s_{\mu}^{k}}{\prod_{l=1, l \neq \mu}^{n}\left(s_{\mu}-s_{l}\right)^{\kappa_{l}}}\right) .
$$

The assertion of the lemma follows by substituting (A.8) into (A.7) and interpreting $\left(s-s_{\mu}\right)^{-j}$ as $\left\{x^{j-1} \frac{\exp \left(x s_{\mu}\right)}{(j-1) !}\right\}$, according to equation (A.1).

\section{REFERENCES}

[1] M. Fliess, J. Lévine, Ph. Martin and P. Rouchon, Flatness and defect of non-linear systems: Introductory theory and examples. Internat. J. Control 61 (1995) 1327-1361.

[2] M. Fliess, Ph. Martin, N. Petit and P. Rouchon, Commande de l'équation des télégraphistes et restauration active d'un signal. Traitement du Signal 15 (1998) 619-625.

[3] M. Fliess and H. Mounier, Controllability and observability of linear delay systems: An algebraic approach. ESAIM: COCV 3 (1998) 301-314. (URL: http://www.emath.fr/COCV/).

[4] M. Fliess and H. Mounier, Tracking control and $\pi$-freeness of infinite dimensional linear systems, edited by G. Picci and D.S. Gilliam, Dynamical Systems, Control, Coding, Computer Vision. Birkhäuser (1999) 45-68.

[5] M. Fliess, H. Mounier, P. Rouchon and J. Rudolph, Controllability and motion planning for linear delay systems with an application to a flexible rod, in Proc. 34th IEEE Conference on Decision and Control. New Orleans (1995) 2046-2051.

[6] M. Fliess, H. Mounier, P. Rouchon and J. Rudolph, Systèmes linéaires sur les opérateurs de Mikusiński et commande d'une poutre flexible. ESAIM Proc. 2 (1997) 183-193. (http://www.emath.fr/proc).

[7] M. Fliess, H. Mounier, P. Rouchon and J. Rudolph, Controlling the transient of a chemical reactor: A distributed parameter approach, in Proc. Computational Engineering in Systems Application IMACS Multiconference, (CESA'98). Hammamet, Tunisia (1998).

[8] F. John, Partial Differential Equations, 4th Edition. Springer-Verlag, New York (1991). 
[9] B. Laroche, Ph. Martin and P. Rouchon, Motion planning for the heat equation. Int. J. Robust Nonlinear Control 10 (2000) 629-643.

[10] A.F. Lynch and J. Rudolph, Flachheitsbasierte Randsteuerung parabolischer Systeme mit verteilten Parametern. Automatisierungstechnik 48 (2000) 478-486.

[11] J. Mikusiński, Sur les équations différentielles du calcul opératoire et leurs applications aux équations aux dérivées partielles. Stud. Math. 12 (1951) 227-270.

[12] J. Mikusiński, Operational Calculus, Vol. 1. Pergamon, Oxford \& PWN, Warszawa (1983).

[13] J. Mikusiński and Th.K. Boehme, Operational Calculus, Vol. 2. Pergamon, Oxford \& PWN, Warszawa (1987).

[14] H. Mounier, J. Rudolph, M. Petitot and M. Fliess, A flexible rod as a linear delay system, in Proc. 3rd European Control Conference. Rome, Italy (1995) 3676-3681.

[15] N. Petit and P. Rouchon, Motion planning for heavy chain systems. SIAM J. Control Optim. 40 (2001) 275-495.

[16] N. Petit and P. Rouchon, Dynamics and solutions to some control problems for water-tank systems. IEEE Trans. Automat. Control AC-47 (2002) 594-609.

[17] I.G. Petrovskij, Über das Cauchysche Problem für Systeme von partiellen Differentialgleichungen. Mat. Sb. 2 (1937) $815-866$.

[18] R. Rothfuß, J. Rudolph and M. Zeitz, Flachheit: Ein neuer Zugang zur Steuerung und Regelung nichtlinearer Systeme. Automatisierungstechnik 45 (1997) 517-525.

[19] W. Rudin, Real and Complex Analysis, 3rd Edition. McGraw-Hill (1987).

[20] J. Rudolph, Randsteuerung von Wärmetauschern mit örtlich verteilten Parametern: Ein flachheitsbasierter Zugang. Automatisierungstechnik 48 (2000) 399-406.

[21] J. Rudolph and F. Woittennek, Flachheitsbasierte Steuerung eines Timoshenko-Balkens. Z. Angew. Math. Mech. 83 (2003) 119-127.

[22] J.C. Simo, A finite strain beam formulation. The three-dimensional dynamic problem. Part one. Comp. Meths. Appl. Mech. 49 (1985) 55-70.

[23] K. Yosida, Operational Calculus. Springer-Verlag (1984).

[24] K. Yuan, Control of slew maneuver of a flexible beam mounted non-radially on a rigid hub: A geometrically exact modelling approach, Vol. 204 (1997) 795-806. 